\title{
1 The draft genome sequence of Japanese rhinoceros beetle
}

\section{Trypoxylus dichotomus}

3

4 Authors:

5 Shinichi Morita ${ }^{1}$, Tomoko F. Shibata ${ }^{2}$, Tomoaki Nishiyama ${ }^{3}$, Yuuki Kobayashi ${ }^{4}$, Katsushi

6 Yamaguchi $^{5}$, Kouhei Toga $^{6,7}$, Takahiro Ohde ${ }^{1,6,8}$, Hiroki Gotoh ${ }^{6,9}$, Takaaki Kojima ${ }^{10}$, Jesse

7 Weber $^{11}$, Marco Salvemini ${ }^{12}$, Takahiro Bino $^{5}$, Mutsuki Mase ${ }^{1,6}$, Moe Nakata ${ }^{6}$, Tomoko Mori ${ }^{5}$,

8 Shogo Mori $^{5}$, Richard Cornette ${ }^{13}$, Kazuki Sakura ${ }^{1}$, Laura C. Lavine ${ }^{14}$, Douglas J. Emlen ${ }^{15}$,

9 Teruyuki Niimi ${ }^{1,6.16 *}$ and Shuji Shigenobu ${ }^{4,5,16 *}$

10

11 Affiliations:

12 1) Division of Evolutionary Developmental Biology, National Institute for Basic Biology, Okazaki, 13 Japan

14 2) Division of Evolutionary Biology, National Institute for Basic Biology, Okazaki, Japan

15

16 
25 9) Department of Biological Science, Faculty of Science, Shizuoka University, Shizuoka, Japan

26 10) Laboratory of Molecular Biotechnology, Graduate School of Bioagricultural Sciences,

27 Nagoya University, Nagoya, Japan

28 11) Department of Integrative Biology, University of Wisconsin-Madison, Madison, Wisconsin,

29 United states of America

30 12) Department of Biology, University of Naples Federico II, Via Cinthia, 80126, Italy

31 13) Institute of Agrobiological Sciences, National Agriculture and Food Research Organization,

32 Tsukuba, Japan

33 14) Department of Entomology, Washington State University, Pullman, Washington, United

34 states of America

35 15) Division of Biological Sciences, The University of Montana, Missoula, Montana, United

36 states of America

37 16) Department of Basic Biology, School of Life Science, The Graduate University for Advanced

38 Studies, SOKENDAI, Okazaki, Japan

39

40 *corresponding authors: Shuji Shigenobu <shige@nibb.ac.jp>, Teruyuki Niimi

41 <niimi@nibb.ac.jp> 


\section{Abstract}

43 Beetles are the largest insect order and one of the most successful animal groups in terms of

44 number of species. The Japanese rhinoceros beetle Trypoxylus dichotomus (Coleoptera,

45 Scarabaeidae, Dynastini) is a giant beetle with distinctive exaggerated horns present on the

46 head and prothoracic regions of the male. T. dichotomus has been used as research model in

47 various fields such as evolutionary developmental biology, ecology, ethology, biomimetics, and

48 drug discovery. In this study, de novo assembly of $615 \mathrm{Mb}$, representing $80 \%$ of the genome

49 estimated by flow cytometry, was obtained using the 10x Chromium platform. The scaffold N50

50 length of the genome assembly was $8.02 \mathrm{Mb}$, with repetitive elements predicted to comprise

$5149.5 \%$ of the assembly. In total, 23,987 protein-coding genes were predicted in the genome. In

52 addition, de novo assembly of the mitochondrial genome yielded a contig of 20,217 bp. We also

53 analyzed the transcriptome by generating 16 RNA-seq libraries from a variety of tissues of both

54 sexes and developmental stages, which allowed us to identify 13 co-expressed gene modules.

55 The detailed genomic and transcriptomic information of $T$. dichotomus is the most comprehensive among those reported for any species of Dynastinae. This genomic information

57 will be an excellent resource for further functional and evolutionary analyses, including the

58 evolutionary origin and genetic regulation of beetle horns and the molecular mechanisms

59 underlying sexual dimorphism.

60

61 Key words: Coleoptera, Trypoxylus dichotomus, horned beetle, draft genome sequence,

62 mitochondrial genome 


\section{Introduction}

64 Beetles (Insecta: Coleoptera) are the largest order not only among insects but among all animal,

65 and they are regarded as one of the most successful animal groups. ${ }^{1}$ Beetles exhibit

66 extraordinary morphological, ecological, and behavioral diversity ${ }^{2}$ and have been used as

67 models to study ecological and evolutionary biology for centuries. For example, when Darwin

68 first introduced the term "sexual selection," the rhinoceros beetle horn was featured as a typical 69 example. $^{3}$

Trypoxylus dichotomus, commonly known as the Japanese rhinoceros beetle, is a giant beetle reaching up to $91.7 \mathrm{~mm}$ in length (Fig. 1a). First described by Linnaeus, ${ }^{4}$ T. dichotomus belongs to the Scarabaeidae, Dynastini, Trypoxylus. The genus Trypoxylus is most closely related to the genus Xyloscaptes (Fig. 1b). ${ }^{1,5-8}$ T. dichotomus inhabits East Asia including Japan, China, Tibet, Taiwan, the Korean peninsula, Northeastern India, Thailand, Vietnam, Laos, and Myanmar. T. dichotomus is characterized by the set of exaggerated horns present in

77 the head and prothoracic regions of males, while females have no horns. The horn of the head region (head horn) can extend to more than 2/3 of the male's body length and is bifurcated twice at the distal tip. The horn of the prothoracic region (thoracic horn) is shorter and bifurcated once at the distal tip. Both horns are strongly sexually dimorphic, and are used as weapons in combat between males over feeding territories visited by females. ${ }^{9}$ Battles take place on the trunks of host trees, with males inserting their head horn under the prothorax of an opponent and attempting to scoop the rival off of the tree. Biomechanical studies of horn morphology indicate

84 that the shape of the horn, particularly the triangular cross-section of the base of the horn,

85 resists buckling when twisted and is well suited to the nature of the battles in this species. ${ }^{10}$ 
88 a mixture of fecal pellets and humus to pupate below ground. In early summer, the adults

89 appear in broad-leaved forests to ingest sap, and males and females mate on trees near

90 feeding sites. After mating, the female lays the eggs in the humus. Adults have a maximum

91 lifespan of about three months and are unable to overwinter.

92

T. dichotomus is an emerging model insect with many advantages. First, it is easy to

94 obtain and the breeding / culturing system in the laboratory has been well developed. Since $T$.

95 dichotomus is a popular pet in Japan, larvae are commercially available for approximately 1

96 USD each, and related items necessary for breeding (e.g., breeding cages, food for larvae and

97 adults, artificial pupal chambers, etc.) can be purchased at a low cost. Larvae are easily

98 maintained in the laboratory and can be stored at low temperatures to delay the onset of

99 pupation, facilitating their use for research throughout the year. ${ }^{11}$ For the critical period from

100 prepupa to adult, when horn formation occurs, a soil-free breeding system has been established

101 that permits non-invasive continuous monitoring and the precise staging of horn development. ${ }^{12}$

102 Second, an RNA interference (RNAi) technique has been established in T. dichotomus, which

103 allows functional assessment of genes of interest. ${ }^{11-16}$ Larval RNAi is performed by injecting

104 double-stranded RNA (dsRNA) into the 1st thoracic segment (T1 segment) of the last instar

105 larva just before the prepupal stage. Larval RNAi is so efficient that researchers can carry out

106 large-scale RNAi screens against candidate genes, as proved by our successful identification of

107 genes related to horn formation. ${ }^{11-16}$ Third, the large body size of $T$. dichotomus has advantages

108 in efficient sampling for various experiments, which could facilitate biochemical assays and

109 molecular analyses including next-generation sequencing (NGS). ${ }^{15,17}$ For example, sufficient

110 amounts of RNA and DNA required for NGS studies can be obtained from each tissue of a

111 single individual alone as shown in this paper. 
Studies of $T$. dichotomus have increased rapidly in a wide variety of research fields due

114 to the utility of this species as a model system. For example, in developmental biology the

115 mechanisms of $T$. dichotomus horn formation have been described in depth. ${ }^{18}$ The molecular

116 pathways underlying sexual dimorphism of the exaggerated horns have been intensively

117 studied, ${ }^{11,12}$ and numerous genes involved in horn formation have been identified by

118 comprehensive transcriptome analyses. ${ }^{15}$ In addition, beetle horns are considered to be an

119 evolutionary novelty because there is no obvious homologous structure in the ancestral

species, ${ }^{19}$ and $T$. dichotomus horns have proven to be an excellent model for exploring how

121 novel morphological structures arise.

The length of $T$. dichotomus horns is exquisitely sensitive to the nutritional condition of

larvae. ${ }^{20}$ Although many exaggerated ornaments and weapons of sexual selection exhibit such

nutrition- or condition-dependent expression - indeed, this plasticity is considered to be an

integral component of their function as a reliable signal of the quality of a male -- the horns of $T$.

dichotomus were the first sexually-selected structure of any animal species to have these

underlying mechanisms of conditional expression explored at a developmental or genetic

level. ${ }^{13}$ The resulting variation in male horn length is mildly polyphenic, yielding major and minor males,,$^{9,21}$ and ethological studies revealed that males tap each other with their head

130 horns before direct combat, ${ }^{22}$ to assess the size of their competitors and avoid unnecessary

131 fighting. ${ }^{23}$ Less competitive minor males have alternative reproductive strategies to spend more 132 time mating with females ${ }^{24}$ and appear at feeding sites earlier than major males to avoid fighting 133 and encounter females. ${ }^{25,26}$

The large size and extraordinary morphology of $T$. dichotomus has inspired several

135 biomimetic studies. For example, the mechanical properties of elytra (fore wings) ${ }^{27-30}$ and the

136 aerodynamic mechanisms ${ }^{31}$ of $T$. dichotomus flight have been studied and applied to industrial

137 uses. In addition, an anti-bacterial peptide ${ }^{32-38}$ and a molecule with anti-prion activity ${ }^{39}$ were

138 discovered from $T$. dichotomus and medical applications of these molecules are expected. 
Thus, $T$. dichotomus has been actively used in various research fields, including evolutionary developmental biology, ecology, ethology, biomimetics, and drug discovery.

144 In the present study, we present the draft genome of T. dichotomus, together with the functional

145 annotation and gene expression data. In addition, we compared the gene repertoire of $T$.

146 dichotomus with those of three other insects. This genomic information provides a crucial

147 foundation for future studies using $T$. dichotomus as well as the comparative genomics of 148 insects.

\section{Materials and Methods}

\section{Insects}

152 We purchased T. dichotomus larvae from Loiinne (Gunma, Japan). Last (third) instar larvae

153 were sexed as described previously, ${ }^{11}$ individually fed on humus in plastic containers, and kept 154 at $10^{\circ} \mathrm{C}$ until use. For tissue sampling, larvae were moved to room temperature for a minimum 155 of 10 days and reared at $28^{\circ} \mathrm{C}$.

\section{Genome sequencing and de novo assembly}

157 Male leg primordia derived from a single individual at $72 \mathrm{~h}$ after pupal-chamber formation, which

158 is before cuticle pigmentation and sclerotization, were dissected out from prepupa in $0.75 \%$

159 sodium chloride solution, and used for genome analysis. 
generated by spraying the buffer into liquid nitrogen in a glass beaker, was added to the sample and blended quickly. Letting the mixture thaw in a tube, RNaseA (QIAGEN, Cat \#19101) and Proteinase K (QIAGEN, Cat \#1019499) were added, and the sample was incubated at room

166 temperature for $2.5 \mathrm{~h}$ without agitation. The sample was centrifuged at $5000 \mathrm{x} \mathrm{g}$ for $30 \mathrm{~min}$ and

167 the supernatant was subjected to DNA extraction with a QIAGEN Genomic-tip 100/G column 168 (Cat \# 10243). The genomic DNA was eluted with $5 \mathrm{ml}$ of Buffer QF, and then purified and concentrated using 0.5-fold volume of Agencourt AMPure XP magnetic beads (Beckman Coulter, Brea, CA, USA, Cat \# A63881) yielding $12.2 \mu \mathrm{g}$ of DNA (174.0 ng/ $\mu \mathrm{l})$. The DNA was size-fractioned by using SAGE HLS (Sage Science, Beverly, MA, USA, Cat \#HLS0001), and the fraction with the highest molecular size was collected. The size distribution of the HMW DNA was evaluated by pulsed-field-gel-electrophoresis (PFGE). In brief, 20 ng of HMW DNA was run on a $1 \%$ agarose gel (Seakem Gold Agarose, Lonza, Rockland, ME, USA, Cat \#50150) in had an approximate mean size of 50 - $80 \mathrm{kbp}$, while shorter DNA fragments (< 10 kbp) were efficiently removed. DNA concentration was measured with a Qubit fluorometer (Thermo Fisher Scientific, Cat \#Q32866) using the Qubit ${ }^{\mathrm{TM}}$ dsDNA BR Assay Kit (Thermo Fisher Scientific, Cat $182 \#$ \#32850).

We constructed a 10x Genomics Chromium linked-read library using 0.68 ng of the 184 HMW DNA extracted as described above with a Chromium Genome Library Kit \& Gel Bead Kit 185 v2 (10x Genomics, San Francisco, CA, USA, Cat \#120258) following the manufacturer's 186 protocol. The generated library was sequenced on a HiSeqX (Ilumina, San Diego, CA, USA) at 187 Macrogen Japan Corp (Tokyo, Japan). The obtained Illumina reads were assembled using the 188 Supernova assembler (ver. 2.1.0) ${ }^{41}$ with a parameter of maxreads $=265000000$. 
We assembled the mitochondrial genome of $T$. dichotomus separately from a paired-end library. Genomic DNA was extracted from the leg primordia using the QIAGEN Genomic-Tip. A paired-end library was prepared with the TruSeq DNA PCR-Free Library Preparation Kit

192 (Illumina) from $1 \mu \mathrm{g}$ of the genomic DNA. The library was sequenced using the Illumina HiSeqX 193 system with $2 \times 150$ bp paired-end sequencing protocol at Macrogen Japan Corp (Tokyo, 194 Japan), where 182,102,568 read-pairs were produced. The mitochondrial genome was 195 assembled using a subset of the raw reads (1 M reads, $302 \mathrm{Mb}$ ) by NovoPlasty ( $\mathrm{v} 4.3 .1)^{42}$ with a parameter of K-mer $=33$, using a partial sequence of $16 \mathrm{~S}$ rRNA of $T$. dichotomus (GenBank accession: $A B 178318.1$ ) as the seed sequence. Our de novo assembly yielded two contigs, Contig1 (20,217 bp) and Contig2 (1,163 bp). A manual inspection revealed that Contig2 was a repetitive sequence included in Contig1 and Contig1 corresponds to the mitochondrial genome. Note that the repetitive regions were not fully resolved by the de novo assembly from Illumina contig was annotated using MITOS2 webserver (http: //mitos2.bioinf.uni-leipzig.de) ${ }^{43}$ with the invertebrate mitochondrial genetic code.

\section{Genome annotation}

205 Repeat annotation: We analyzed the distribution of repetitive elements in the genome of $T$. 206 dichotomus, using RepeatModeler (ver. open-1.0.8) ${ }^{44}$ and RepeatMasker (ver. open-4.0.6) ${ }^{45}$. Gene prediction: We annotated the $T$. dichotomus genome for protein-coding genes using RNA-seq data. We generated 16 libraries covering 6 tissues and 6 developmental time points from early embryogenesis to postembryonic stages (see RNA-Seq analysis section below). The Illumina RNA-seq reads were aligned against a hard-masked genome in which

211 genomic repetitive elements were substituted by ' $N$ ', using HISAT2 (ver. 2.1.0) ${ }^{46}$ with default 212 parameters. The BRAKER2 (ver. 2.1.5) ${ }^{47}$ pipeline was used to predict protein-coding genes 213 based on the RNA-seq alignments. 
Gene annotation: All predicted protein-coding genes were compared with the NCBI non-

215 redundant protein database (nr DB, release November 2020) using the blastp command of

216 diamond software (ver. 2.0.5) ${ }^{48}$ with a threshold of e-value $<1.0 \mathrm{e}-5$. We used InterProScan (ver.

$2175.48)^{49}$ to query the predicted coding regions for known functional domains and assign Gene

218 Ontology (GO) terms to the proteins. We also used the eggNOG-mapper pipeline (ver. 2.0.1b)

219 for functional annotation (including GO term assignments) of the predicted genes based on the

220 orthology information.

221 BUSCO (ver. 4.0.6 $)^{51}$ was used in quantitative measuring for the assessment of

222 genome assembly, using insecta_odb10 (1367 total orthogroups) as the lineage input. A

223 genome browser was built using JBrowse2 web (ver. 1.5.1) ${ }^{52}$ and is available at

224 http://www.insect.nibb.info/trydi/.

\section{Ortholog analysis}

227 We used the OrthoFinder (ver. 2.3.11) ${ }^{53}$ to generate clusters of orthologous and paralogous 228 gene families. Public gene datasets of Onthophagus taurus (RefSeq accession No.

229 GCF_000648695), Tribolium castaneum (UniProt accession No. UP000007266), and

230 Drosophila melanogaster (UniProt accession No. UP000000803) were used as references.

\section{RNA-Seq analysis}

233 In total, 16 samples (egg, non-sexed embryos from five stages, ovary, testis, and Malpighian 234 tubule, hindgut, brain and fat body of males and females respectively (Table S1)) were used for 235 RNA-seq analysis. Eggs, ovaries, and testes were dissected out from adult insects in $0.75 \%$ 236 sodium chloride solution. Malpighian tubule, hindgut, brain and fat body were dissected out from 
237 third instar larvae in $0.75 \%$ sodium chloride solution. These samples were frozen in liquid

238 nitrogen and stored at $-80^{\circ} \mathrm{C}$ until use.

Total RNA was extracted from each tissue sample, except for the fat body sample, using the RNeasy Micro Kit (QIAGEN, Cat \# 74004) according to the manufacturer's instructions.

241 Total RNA of the fat bodies was extracted using TRIzol Reagent (Thermo Fisher Scientific, Cat

242 \#15596-026) according to the manufacturer's instructions. These total RNAs were treated with

243 DNase using RNase-Free DNase Set (QIAGEN, Cat \#79254), from which Illumina sequencing

244 libraries were prepared using the TruSeq Stranded mRNA library prep kit (llumina) following the

245 manufacturer's instructions. The libraries were multiplexed and sequenced using the Illumina

246 HiSeq1500 system with 2 x 101 bp paired-end sequencing protocol at the Functional Genomics

247 Facility of National Institute for Basic Biology.

T. dichotomus RNA-Seq reads generated from these 16 libraries were adapter-trimmed

249 using Trim Galore! (ver. 0.5.0) $)^{54}$ and cutadapt (ver. 1.18) ${ }^{55}$. The cleaned RNA-Seq reads were

250 mapped to the T. dichotomus genome assembly using HISAT2 (ver. 2.1.0) ${ }^{46}$ with default

251 parameters. For quantification of the gene expression, StringTie (ver. 2.1.3) ${ }^{56}$ and prepDE.py

252 (http://ccb.jhu.edu/software/stringtie/dl/prepDE.py) with a GTF file of the BRAKER2 gene

253 prediction were used to generate the count matrix. The count data were normalized by the

254 trimmed mean $M$ values (TMM) method $^{57}$ available in the edgeR library (ver. 3.32.1 $)^{58}$. To

255 visualize profiles of gene expression, a multidimensional scaling (MDS) plot was generated

256 using the edgeR software package.

257 To detect modules of co-expressed genes from the transcriptome data, Weighted Gene

258 Correlation Network Analysis (WGCNA) was applied. ${ }^{59}$ Normalized count data were used for

259 this analysis implemented in the WGCNA library (version 1.70) ${ }^{60}$. Genes expressed at low

260 levels, and genes with low expression variance across the libraries, were filtered out; the 6666

261 surviving gene models were used in the WGCNA analysis. A signed network was constructed in 
WGCNA with specific parameter settings of power = 8, networkType="signed", TOMType="unsigned", minModuleSize=30.

\section{Genome size estimation using flow cytometry}

266 Flow cytometry estimates were made by quantification of fluorescence from propidium iodide

267 (PI) stained nuclei extracted from primordia of horns, legs or wings of male pupae with the 268 fruitfly, Drosophila melanogaster $(1 \mathrm{C}=173.3 \mathrm{Mbp})$ as an internal standard. Samples were 269 added to $1 \mathrm{~mL}$ of PBS and homogenized with BioMasher II (nippi, Tokyo, Japan, cat\# 320102), 270 and then $1 \mu$ l of $10 \%$ Triton-X (SIGMA-ALDRICH, St Louis, MO, USA, cat\# 93443) and $4 \mu$ of $271100 \mathrm{mg} / \mathrm{ml}$ RNase A (QIAGEN, cat\# 19101) were added to the homogenate. The resultant 272 solution was passed through a $30 \mu \mathrm{m}$ CellTrics filter (Sysmex Partec, Görlitz, Germany, cat\# 273 BP486257) and stained with $10 \mu \mathrm{g} / \mathrm{ml} \mathrm{PI} \mathrm{(Sony} \mathrm{Biotechnology,} \mathrm{San} \mathrm{Jose,} \mathrm{CA,} \mathrm{USA,} \mathrm{cat \#}$ 274 2706505). The nuclei were analyzed on a Cell Sorter SH800 (SONY Biotechnology) for three 275 replicates.

\section{Genome size estimation from reads of shotgun sequencing}

278 Genomic DNA was extracted from the leg primordia using the QIAGEN Genomic-Tip (Cat \# 279 10243). A paired-end library was prepared with the TruSeq DNA PCR-Free LT Sample 280 Preparation Kit (Illumina, \#FC-121-3001) from $1 \mu \mathrm{g}$ of the genomic DNA. The library was 281 sequenced using the Illumina HiSeqX system with $2 \times 150$ bp paired-end sequencing protocol at 282 Macrogen Japan Corp (Tokyo, Japan), where 182,102,568 read-pairs were produced. To 283 estimate the genome size of $T$. dichotomus, we analyzed the distribution of k-mers from the 284 Illumina reads using Jellyfish (ver. 2.2.10) ${ }^{61}$ and GenomeScope 2.0 (git commit id: fdeb891) ${ }^{62}$. 285 The distribution of kmers of size was analyzed with three different kmer sizes, 21, 31 and 41. 


\section{Data availability}

288 Data from whole-genome sequencing and transcriptome sequencing have been deposited in 289 the DDBJ database under BioProject accession PRJDB12657. The analyzed data including 290 genome assembly, gene prediction, annotation, and gene expression are available through 291 FigShare (https://doi.org/10.6084/m9.figshare.c.5737754). The genome browser is available at 292 http://www.insect.nibb.info/trydi/.

\section{Results and Discussion}

\section{Genome size estimate}

296 Using flow cytometry and the fruitfly Drosophila melanogaster as reference, we determined that 297 the haploid genome size of $T$. dichotomus is $773.1 \pm 24.6 \mathrm{Mb}$. In addition, we estimated the $T$. 298 dichotomus genome size from the distribution of k-mers from Illumina reads of shotgun 299 sequences. The distribution of kmers of size 21, 31 and 41 resulted in an estimated haploid 300 genome size of 710-764 Mb. The small discrepancy between k-mer and cytometry-based 301 estimates may be caused by the repetitive elements (see below), which can affect k-mer 302 estimates.

\section{Genome assembly and evaluation}

304 Genome sequencing of $T$. dichotomus was performed with genomic DNA isolated from leg 305 primordia of a single male prepupa collected in Gunma, Japan. We prepared high molecular 306 weight (HMW) DNA from the beetle sample with an approximate mean size of 50 - $80 \mathrm{kbp}$. The 307 HMW genome DNA (0.68 ng) was subjected to the linked-read whole-genome sequencing 308 library construction on the 10x Chromium platform. A draft genome was assembled with 
Supernova ${ }^{41}$ using $265.02 \mathrm{M}$ Illumina reads (57.0 x coverage). The haploid genome assembly was named TdicSN1.0 and used for downstream analysis.

The final assembly TdicSn1.0 consists of 15,609 scaffolds with an N50 of $8.02 \mathrm{Mb}$ and a

total size of $615 \mathrm{Mb}$ [Table 1], covering $80 \%$ of the genome. We evaluated the completeness of the assembly by using the benchmarking universal single-copy orthologs (BUSCO). ${ }^{63}$ The BUSCO analysis showed that our $T$. dichotomus genome assembly has high coverage of coding regions, capturing $99.4 \%$ (98.9\% complete, $0.5 \%$ fragmented) from the Insecta dataset (version 4.0.6; $n=1,367$ ) [Table 2]. The score is comparable to the genome of the model beetle

Tribolium castaneum. ${ }^{64}$

We assembled the mitochondrial genome separately from a paired-end library using a known partial sequence of $16 \mathrm{~S}$ rRNA of $T$. dichotomus (GenBank accession: AB178318.1) as the seed sequence. Our de novo assembly yielded a contig of 20,217 bp. We identified 2 ribosomal RNAs, 22 tRNAs, and 13 protein-coding genes. The gene repertoire and the structural arrangement showed typical features of mitochondrial genomes of insects, but the total length of the mitochondrial genome was longer than that of the model coleopteran Tribolium castaneum (15.8 kb (NC_003081.2)) by $4.3 \mathrm{~kb}$. The increased length is mostly due to species specific repetitive elements between the s-rRNA gene and the tRNA-GLN gene.

\section{Genome annotation}

327 The $T$. dichotomus genome is rich in repetitive elements, which total $305 \mathrm{Mb}$ and account for $32849.5 \%$ of the genome assembly [Table S2]. Considering the situation that the current assembly covers $80 \%$ of the estimated genome size, it is likely that uncovered regions contain more repetitive sequences that are generally difficult to capture by Illumina-based genome assembling. We annotated the $T$. dichotomus genome for protein-coding genes using RNA-seq data. Aiming for a comprehensive gene identification, we collected RNA samples from a wide 
334 from 6 tissues (brain, Malpighian tubule, hindgut, fat body, testis and ovary) and from 6 time

335 points of whole embryos from middle to late developmental stages [Table S1]. The alignment

336 data of these RNA-seq sequences mapped on the TdicSN1.0 assembly were subjected to the

337 BRAKER2 pipeline to predict protein-coding genes. In total, 23,987 protein-coding genes are

338 predicted in the $T$. dichotomus genome. Of these, 19,708 (82.6\%) encoded proteins exhibiting

339 sequence similarity to proteins in the NCBI non-redundant protein database. The two most

340 frequent top-hit species corresponded to scarab beetles, Oryctes borbonicus, $(6,312)$ and

341 Onthophagus taurus $(5,126)$, followed by other Coleoptera species such as Tribolium

342 castaneum, Ignelater luminosus, and Nicrophorus vespilloides, which reasonably reflects the

343 phylogenetic position of $T$. dichotomus [Fig $1 \mathrm{~b}$ ]. We used InterProScan to query the predicted

344 coding regions for known functional domains. We identified 29,549 Pfam motifs ${ }^{65}$ and 34,594

345 PANTHER motifs ${ }^{66}$ in the products of 14,497 and 15,139 T. dichotomus gene models,

346 respectively. We also identified 2,389 proteins with secretion potential predicted by the SignalP

347 program $^{67}$. By integrating protein-domain based and orthology-based approaches using

348 InterProScan and eggNOG-mapper pipelines, 581,644 Gene Ontology terms were assigned to

$349 \quad 10,541$ genes (43.9\%).

\section{Ortholog analysis}

352 To understand the gene repertoire evolution of $T$. dichotomus, we generated clusters of

353 orthologous and paralogous gene families comparing the $T$. dichotomus proteome with those of

354 three other insects, Onthophagus taurus, Tribolium castaneum and Drosophila melanogaster.

355 O. taurus is a dung beetle belonging to the family Scarabaeidae [Fig. 1b], and represents

356 another model insect for the study of horn polyphenism. ${ }^{68}$ We included T. castaneum as a

357 model coleoptera and D. melanogaster as a model insect for comparison as outgroups. The

358 OrthoFinder program identified 12,034 orthogroups consisting of 56,656 genes derived from all 
four insects. The 18,783 T. dichotomus gene models were clustered into 10,291 orthogroups, leaving 5,204 genes unassigned to any orthogroups (i.e., orphan genes). Among them, 8,219 groups were shared within three Coleoptera species, while 543 groups were shared within only the horned beetles, O. taurus and T. dichotomus [Fig. 2a]. These beetle-specific groups may account for the common characteristic traits such as the elytron of the beetle ${ }^{69}$ and the exaggerated horns of $T$. dichotomus and O. taurus. We found 967 groups, consisting of 5,705 genes, that are unique to $T$. dichotomus and may account for lineage specific traits [Fig. 2a].

\section{Transcriptome analysis}

We analyzed 16 RNA-seq libraries created from a variety of tissues of both sexes and developmental stages [Table S1]. We quantified gene expression and profiled the expression patterns across all of the sample tissues. The multidimensional scaling (MDS) plot of the 16 samples depict the transcriptome similarities among the samples [Fig. 3a]. Samples derived from the same organs clustered together irrespective of sex differences. Eggs and ovaries also clustered together, as did the transcriptomes of embryos. Notably, embryonic samples were roughly ordered on the plot from earlier to later stages, which may represent the gradual transcriptional progression during embryogenesis of $T$. dichotomus. We performed Weighted Gene Correlation Network Analysis (WGCNA) to understand

377 the co-expression relationship between genes at a system level. WGCNA identified 13 coexpressed modules from the expression data spanning 16 samples; each module contained 71 to 1205 co-expressed genes [Fig. 3bc]. Each module represents genes with highly correlated expression profiles, either in a single organ or in a certain stage of embryogenesis. Out of 13 modules, seven represent organ-specific patterns [Fig. 3bc, module 1 -7]. Both modules 6 and 
sexually differentiated functions of $T$. dichotomus fat body in the sexes. Six modules represent embryonic expression patterns [Fig. 3bc, module 8 -13]. Genes contained in the module 13 exhibited a constant pattern of expression from middle to late stages.

\section{Conclusions}

389 We report the assembly and annotation of the nuclear and mitochondrial genomes of the 390 Japanese rhinoceros beetle $T$. dichotomus. The high completeness and the extensive 391 annotations achieved for this genome, as well as transcriptome data generated from a wide 392 variety of tissues and developmental stages, make this draft assembly an excellent resource for 393 further functional and evolutionary analyses in this emerging model insect For example, the 394 availability of the genome sequence will accelerate the use of functional assays using RNAi and 395 genome editing techniques. These are key tools for experimental investigations of the 396 evolutionary origin and genetic regulation of the exaggerated male horn, and the molecular 397 mechanisms underlying the strong sexual dimorphism in its expression. We also anticipate that 398 recent advances in genome sequencing such as long read sequencing and $\mathrm{Hi}-\mathrm{C}$ proximity 399 ligation methods will allow us extend the existing annotation to include a chromosome-level 400 assembly of $T$. dichotomus in the near future, which would provide us with a more complete 401 picture of the diploid genome composed of 18 autosomes and sex chromosomes (XX/XY). ${ }^{70}$

402 Other research areas such as ethology, biomimetics and drug discovery will benefit from the 403 continuing advancement of genomic resources. Finally, given $T$. dichotomus is one of the most 404 popular insects in Japan, we hope that the genomic resources will facilitate the development of 405 effective population genomic tools to monitor and protect wild populations, which would lead to 406 the establishment of the conservation genomics of the Japanese rhinoceros beetle. These 407 genomic data and a genome browser are available at http://www.insect.nibb.info/trydi/. 


\section{Acknowledgements}

410 We thank Toshiya Ando, Taro Nakamura and Yasuhiko Chikami for helpful discussion, the

411 Model Plant Research Facility/NIBB BioResource Center, the Data Integration and Analysis

412 Facility/NIBB and the Functional Genomics Facility/NIBB Core Research Facilities for technical

413 assistance. This work was supported by MEXT KAKENHI Grant Numbers 23128505, 25128706,

$41416 \mathrm{H} 01452,18 \mathrm{H} 04766,20 \mathrm{H} 04933$ and $20 \mathrm{H} 05944$ (to Te. N.); $17 \mathrm{H} 06384$ (to S.S.); 22128008 (to

415 S.S. and To.N); JSPS KAKENHI Grant Numbers $19 K 16181$ and 21K15135 (to S. M.), and NSF

416 IOS-1456133 (to D. E. and L. L.). 


\section{References}

418 1. Hunt, T., Bergsten, J., Levkanicova, Z., et al. 2007, A Comprehensive Phylogeny of

419 Beetles Reveals the Evolutionary Origins of a Superradiation. Science, 318, 1913-6.

420 2. Crowson, R. A. 1960, The Phylogeny of Coleoptera. Annu. Rev. Entomol., 5, 111-34.

$4213 . \quad$ Darwin Charles. 1871, The descent of man, and selection in relation to sex. London: John

422 Murray. vol. 1.

4234 4inné, C. von. 1771, Mantissa plantarum: Generum editionis VI. Impensis Direct.

424 Laurentii Salvii.

425 5. Ahrens, D., Schwarzer, J., and Vogler, A. P. 2014, The evolution of scarab beetles tracks 426 the sequential rise of angiosperms and mammals. Proc. R. Soc. B Biol. Sci., 281, 20141470.

427 6. Misof, B., Liu, S., Meusemann, K., et al. 2014, Phylogenomics resolves the timing and 428 pattern of insect evolution. Science, 346, 763-7.

$4297 . \quad$ Mckenna, D. D., Wild, A. L., Kanda, K., et al. 2015, The beetle tree of life reveals that 430 Coleoptera survived end-Permian mass extinction to diversify during the Cretaceous terrestrial 431 revolution. Syst. Entomol., 40, 835-80.

432 8. Jin, H., Yonezawa, T., Zhong, Y., Kishino, H., and Hasegawa, M. 2016, Cretaceous 433 origin of giant rhinoceros beetles (Dynastini; Coleoptera) and correlation of their evolution with 434 the Pangean breakup. Genes Genet. Syst., 91, 209-15.

4359 9. Hongo, Y. 2007, Evolution of male dimorphic allometry in a population of the Japanese 436 horned beetle Trypoxylus dichotomus septentrionalis. Behav. Ecol. Sociobiol., 62, 245-53.

437 10. McCullough, E. L., Tobalske, B. W., and Emlen, D. J. 2014, Structural adaptations to 438 diverse fighting styles in sexually selected weapons. Proc. Natl. Acad. Sci., 111, 14484-8.

439 11. Ito, Y., Harigai, A., Nakata, M., et al. 2013, The role of doublesex in the evolution of 440 exaggerated horns in the Japanese rhinoceros beetle. EMBO Rep., 14, 561-7.

441 12. Morita, S., Ando, T., Maeno, A., et al. 2019, Precise staging of beetle horn formation in 442 Trypoxylus dichotomus reveals the pleiotropic roles of doublesex depending on the

443 spatiotemporal developmental contexts. PLOS Genet., 15, e1008063.

444 13. Emlen, D. J., Warren, I. A., Johns, A., Dworkin, I., and Lavine, L. C. 2012, A

445 Mechanism of Extreme Growth and Reliable Signaling in Sexually Selected Ornaments and 446 Weapons. Science, 337, 860-4. 
447 14. Gotoh, H., Hust, J. A., Miura, T., Niimi, T., Emlen, D. J., and Lavine, L. C. 2015, The 448 Fat/Hippo signaling pathway links within-disc morphogen patterning to whole-animal signals 449 during phenotypically plastic growth in insects. Dev. Dyn., 244, 1039-45.

450 15. Ohde, T., Morita, S., Shigenobu, S., et al. 2018, Rhinoceros beetle horn development 451 reveals deep parallels with dung beetles. PLOS Genet., 14, e1007651.

452 16. Adachi, H., Matsuda, K., Niimi, T., Kondo, S., and Gotoh, H. 2020, Genetical control of 453 2D pattern and depth of the primordial furrow that prefigures $3 \mathrm{D}$ shape of the rhinoceros beetle 454 horn. Sci. Rep., 10, 18687.

455 17. Zinna, R., Emlen, D., Lavine, L. C., et al. 2018, Sexual dimorphism and heightened 456 conditional expression in a sexually selected weapon in the Asian rhinoceros beetle. Mol. Ecol., $45727,5049-72$.

458 18. Matsuda, K., Gotoh, H., Tajika, Y., et al. 2017, Complex furrows in a 2D epithelial sheet 459 code the 3D structure of a beetle horn. Sci. Rep., 7, 13939.

460 19. Muller, G. B., and Wagner, G. P. Novelty in Evolution: Restructuring the Concept, 30.

461 20. Emlen, D. J., Lavine, L. C., and Ewen-Campen, B. 2007, On the origin and evolutionary 462 diversification of beetle horns. Proc. Natl. Acad. Sci., 104, 8661-8.

463 21. McCullough, E. L., Ledger, K. J., O’Brien, D. M., and Emlen, D. J. 2015, Variation in 464 the allometry of exaggerated rhinoceros beetle horns. Anim. Behav., 109, 133-40.

465 22. Hongo, Y. 2003, Appraising behaviour during male-male Interaction in the Japanese 466 horned beetle Trypoxylus dichotomus septentrionalis (Kono). Behaviour, 140, 501-17.

467 23. McCullough, E. L., and Zinna, R. A. 2013, Sensilla Density Corresponds to the Regions 468 of the Horn Most Frequently Used During Combat in the Giant Rhinoceros Beetle Trypoxylus 469 dichotomus (Coleoptera: Scarabaeidae: Dynastinae). Ann. Entomol. Soc. Am.

470 24. Karino, K., and Niiyama, H. 2006, Males with short horns spent more time mating in the 471 Japanese horned beetle Allomyrina dichotoma. Acta Ethologica, 9, 95-8.

472 25. Siva-Jothy, M. T. 1987, Mate securing tactics and the cost of fighting in the Japanese 473 horned beetle, Allomyrina dichotoma L. (Scarabaeidae). J. Ethol., 5, 165-72.

474 26. Sol, J. F. del, Hongo, Y., Boisseau, R. P., Berman, G. H., Allen, C. E., and Emlen, D. J. 475 2021, Population differences in the strength of sexual selection match relative weapon size in the 476 Japanese rhinoceros beetle, Trypoxylus dichotomus (Coleoptera: Scarabaeidae) $†$. Evolution, 75, $477 \quad 394-413$. 
478 27. Chen, J., Dai, G., Xu, Y., and Iwamoto, M. 2007, Optimal composite structures in the 479 forewings of beetles. Compos. Struct., 81, 432-7.

480 28. Tuo, W., Chen, J., Wu, Z., Xie, J., and Wang, Y. 2016, Characteristics of the tensile 481 mechanical properties of fresh and dry forewings of beetles. Mater. Sci. Eng. C, 65, 51-8.

482 29. Chen, J., Zhang, X., Okabe, Y., Saito, K., Guo, Z., and Pan, L. 2017, The deformation 483 mode and strengthening mechanism of compression in the beetle elytron plate. Mater. Des., 131, $484 \quad 481-6$.

485 30. Chen, J., Zhang, X., Okabe, Y., Xie, J., and Xu, M. 2019, Beetle elytron plate and the 486 synergistic mechanism of a trabecular-honeycomb core structure. Sci. China Technol. Sci., 62 , $48787-93$.

488 31. Truong, T. V., Le, T. Q., Tran, H. T., Park, H. C., Yoon, K. J., and Byun, D. 2012, Flow 489 visualization of rhinoceros beetle (Trypoxylus dichotomus) in free flight. J. Bionic Eng., 9, 30449014.

491 32. Miyanoshita, A., Hara, S., Sugiyama, M., et al. 1996, Isolation and Characterization of a 492 New Member of the Insect Defensin Family from a Beetle, Allomyrina dichotoma. Biochem. 493 Biophys. Res. Commun., 220, 526-31.

494 33. Saido-Sakanaka, H., Ishibashi, J., Sagisaka, A., Momotani, E., and Yamakawa, M. 1999, 495 Synthesis and characterization of bactericidal oligopeptides designed on the basis of an insect 496 anti-bacterial peptide. Biochem. J., 338, 29-33.

497 34. Saido-Sakanaka, H., Ishibashi, J., Momotani, E., Amano, F., and Yamakawa, M. 2004, In 498 vitro and in vivo activity of antimicrobial peptides synthesized based on the insect defensin. 499 Peptides, 25, 19-27.

500 35. Koyama, Y., Motobu, M., Hikosaka, K., et al. 2006, Cytotoxicity and antigenicity of 501 antimicrobial synthesized peptides derived from the beetle Allomyrina dichotoma defensin in 502 mice. Int. Immunopharmacol., 6, 1748-53.

503 36. Iwasaki, T., Ishibashi, J., Kubo, M., Taylor, D., and Yamakawa, M. 2009, Multiple 504 Functions of Short Synthetic Enantiomeric Peptides Based on Beetle Defensins. Biosci. 505 Biotechnol. Biochem., 73, 683-7.

506 37. Iwasaki, T., Ishibashi, J., Tanaka, H., et al. 2009, Selective cancer cell cytotoxicity of 507 enantiomeric 9-mer peptides derived from beetle defensins depends on negatively charged 508 phosphatidylserine on the cell surface. Peptides, 30, 660-8. 
509 38. Iwasaki, T., Saido-Sakanaka, H., Asaoka, A., Taylor, D., Ishibashi, J., and Yamakawa, 510 M. 2007, In vitro Activity of Diastereomeric Antimicrobial Peptides Alone and in Combination

511 with Antibiotics against Methicillin-resistant Staphylococcus aureus and Pseudomonas

512 aeruginosa. J. Insect Biotechnol. Sericology, 76, 1_25-1_29.

513 39. Hamanaka, T., Nishizawa, K., Sakasegawa, Y., et al. 2015, Anti-prion activity found in

514 beetle grub hemolymph of Trypoxylus dichotomus septentrionalis. Biochem. Biophys. Rep., 3 , $51532-7$.

516 40. Ogata, N. Whole-Genome Sequence of the Trypoxylus dichotomus Japanese rhinoceros 517 beetle. MicroPublication Biol., 2021.

518 41. Weisenfeld, N. I., Kumar, V., Shah, P., Church, D. M., and Jaffe, D. B. 2017, Direct

519 determination of diploid genome sequences. Genome Res., 27, 757-67.

520 42. Dierckxsens, N., Mardulyn, P., and Smits, G. 2016, NOVOPlasty: de novo assembly of 521 organelle genomes from whole genome data. Nucleic Acids Res., gkw955.

522 43. Donath, A., Jühling, F., Al-Arab, M., et al. 2019, Improved annotation of protein-coding 523 genes boundaries in metazoan mitochondrial genomes. Nucleic Acids Res., 47, 10543-52.

524 44. Smit, A., and Hubley, R. 2008, RepeatModeler Open-1.0.

525 45. Smit, A., Hubley, R., and Green, P. 2013, RepeatMasker. Open-4.0.

526 46. Kim, D., Langmead, B., and Salzberg, S. L. 2015, HISAT: a fast spliced aligner with low 527 memory requirements. Nat. Methods, 12, 357-60.

528 47. Brůna, T., Hoff, K. J., Lomsadze, A., Stanke, M., and Borodovsky, M. 2021, BRAKER2: 529 automatic eukaryotic genome annotation with GeneMark-EP+ and AUGUSTUS supported by a 530 protein database. NAR Genomics Bioinforma., 3, lqaa108.

531 48. Buchfink, B., Reuter, K., and Drost, H.-G. 2021, Sensitive protein alignments at tree-of532 life scale using DIAMOND. Nat. Methods, 18, 366-8.

533 49. Blum, M., Chang, H.-Y., Chuguransky, S., et al. 2021, The InterPro protein families and 534 domains database: 20 years on. Nucleic Acids Res., 49, D344-54.

535 50. Huerta-Cepas, J., Forslund, K., Coelho, L. P., et al. 2017, Fast Genome-Wide Functional 536 Annotation through Orthology Assignment by eggNOG-Mapper. Mol. Biol. Evol., 34, 2115-22.

537 51. Seppey, M., Manni, M., and Zdobnov, E. M. 2019, BUSCO: Assessing Genome 538 Assembly and Annotation Completeness In: Kollmar, M., (ed.), Gene Prediction: Methods and 539 Protocols. Springer, New York, NY, pp. 227-45. 
540 52. Skinner, M. E., Uzilov, A. V., Stein, L. D., Mungall, C. J., and Holmes, I. H. 2009,

541 JBrowse: A next-generation genome browser. Genome Res., 19, 1630-8.

542 53. Emms, D. M., and Kelly, S. 2015, OrthoFinder: solving fundamental biases in whole

543 genome comparisons dramatically improves orthogroup inference accuracy. Genome Biol., 16, 544157.

545 54. Krueger, F. 2018, Trim Galore!

546 55. Martin, M. 2011, Cutadapt removes adapter sequences from high-throughput sequencing 547 reads. EMBnet.journal, 17, 10-2.

548 56. Pertea, M., Pertea, G. M., Antonescu, C. M., Chang, T.-C., Mendell, J. T., and Salzberg,

549 S. L. 2015, StringTie enables improved reconstruction of a transcriptome from RNA-seq reads.

550 Nat. Biotechnol., 33, 290-5.

551 57. Robinson, M. D., and Oshlack, A. 2010, A scaling normalization method for differential 552 expression analysis of RNA-seq data. Genome Biol., 11, R25.

553 58. Robinson, M. D., McCarthy, D. J., and Smyth, G. K. 2010, edgeR: a Bioconductor 554 package for differential expression analysis of digital gene expression data. Bioinformatics, 26, $555 \quad 139-40$.

556 59. Zhang, B., and Horvath, S. 2005, A General Framework for Weighted Gene Co557 Expression Network Analysis. Stat. Appl. Genet. Mol. Biol., 4.

558 60. Langfelder, P., and Horvath, S. 2008, WGCNA: an R package for weighted correlation 559 network analysis. BMC Bioinformatics, 9, 559.

560 61. Marçais, G., and Kingsford, C. 2011, A fast, lock-free approach for efficient parallel 561 counting of occurrences of k-mers. Bioinformatics, 27, 764-70.

562 62. Ranallo-Benavidez, T. R., Jaron, K. S., and Schatz, M. C. 2020, GenomeScope 2.0 and 563 Smudgeplot for reference-free profiling of polyploid genomes. Nat. Commun., 11, 1432.

564 63. Simão, F. A., Waterhouse, R. M., Ioannidis, P., Kriventseva, E. V., and Zdobnov, E. M. 565 2015, BUSCO: assessing genome assembly and annotation completeness with single-copy 566 orthologs. Bioinformatics, 31, 3210-2.

567 64. Herndon, N., Shelton, J., Gerischer, L., et al. 2020, Enhanced genome assembly and a 568 new official gene set for Tribolium castaneum. BMC Genomics, 21, 47.

569 65. Mistry, J., Chuguransky, S., Williams, L., et al. 2021, Pfam: The protein families 570 database in 2021. Nucleic Acids Res., 49, D412-9. 
571 66. Mi, H., Ebert, D., Muruganujan, A., et al. 2021, PANTHER version 16: a revised family

572 classification, tree-based classification tool, enhancer regions and extensive API. Nucleic Acids

573 Res., 49, D394-403.

574 67. Nielsen, H., Engelbrecht, J., Brunak, S., and von Heijne, G. 1997, Identification of

575 prokaryotic and eukaryotic signal peptides and prediction of their cleavage sites. Protein Eng.,

576 10, 1-6.

577 68. Moczek, A. P., and Kijimoto, T. 2014, Development and evolution of insect

578 polyphenisms: novel insights through the study of sex determination mechanisms. Curr. Opin.

579 Insect Sci., 1, 52-8.

580 69. Grimaldi, D., Engel, M. S., Engel, M. S., and Engel, S. C. and P. M. S. 2005, Evolution of

581 the Insects. Cambridge University Press.

582 70. Dutrillaux, A.-M., Mamuris, Z., and Dutrillaux, B. 2013, Chromosome analyses

583 challenge the taxonomic position of Augosoma centaurus Fabricius, 1775 (Coleoptera:

584 Scarabaeidae: Dynastinae) and the separation of Dynastini and Oryctini. Zoosystema, 35, 537-

58549. 


\section{Figures}

589 Figure 1. Photograph and phylogenetic context of the Japanese rhinoceros beetle, Trypoxylus

590 dichotomus. (a) Photograph of an adult male (left) and an adult female (right) of $T$. dichotomus.

591 (b) A phylogenetic tree of beetles depicting the phylogenetic relationship between $T$. dichotomus

592 and related beetles with Drosophila melanogaster as an outgroup. Estimated divergence dates

593 (mya: million years ago) are based on Hunt et al., 2007, Ahrens et al., 2014, Misof et al., 2014,

594 Mckenna et al., 2015 and Jin et al., 2016..$^{1,5-8}$
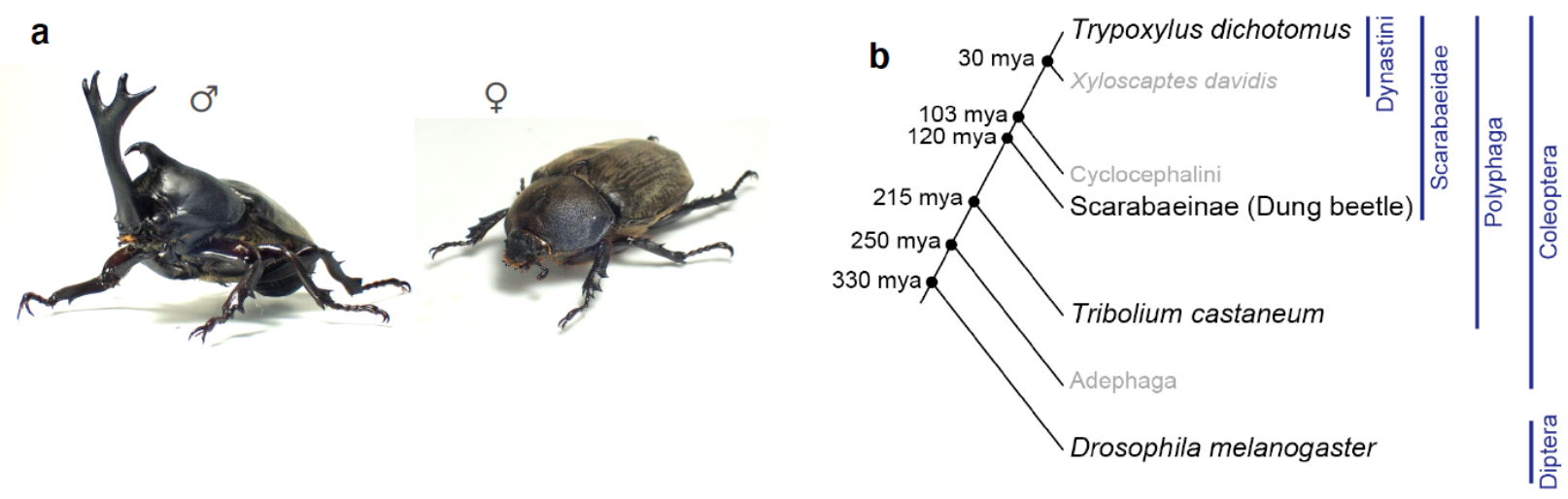
595 Figure 2. Venn diagram of shared and unique orthogroups in four insects. Orthogroups were

596 identified by clustering of orthologous groups using OrthoFinder. ${ }^{53}$

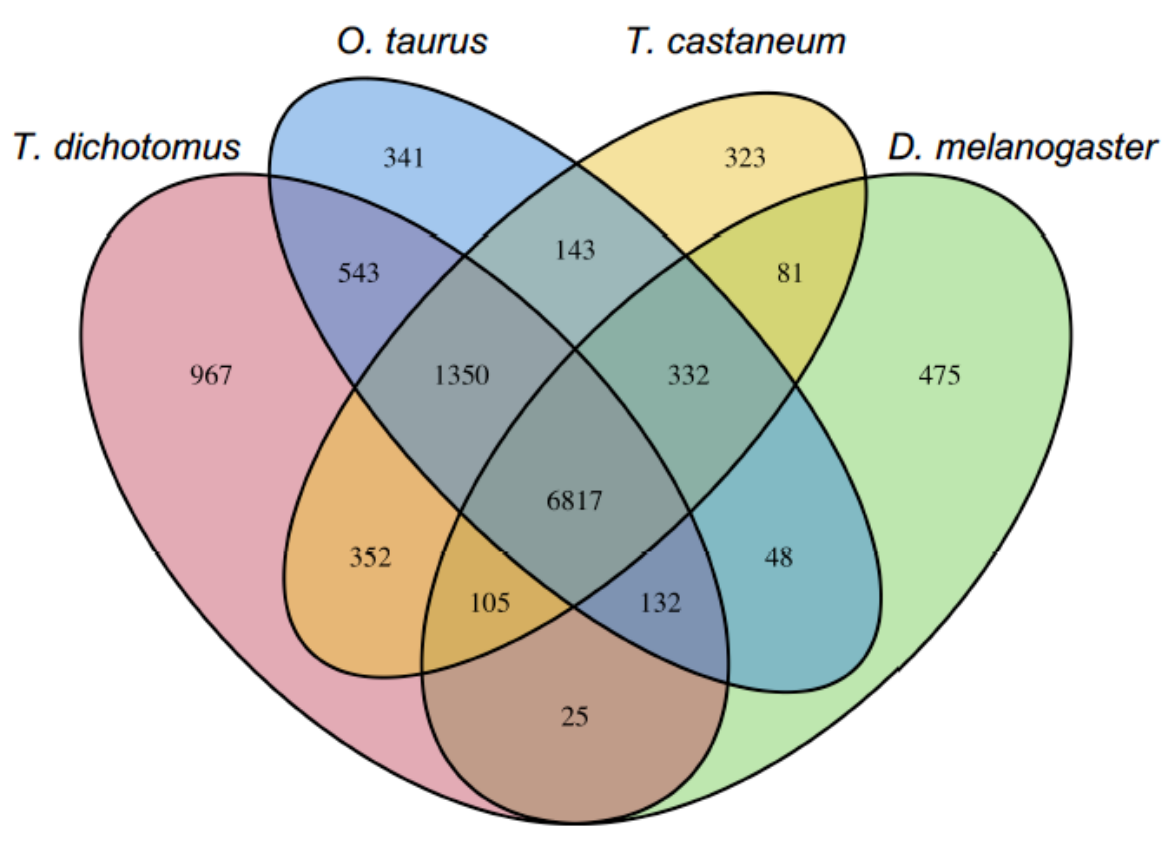


597 Figure 3. Transcriptome analysis. (a) MDS plot for RNA-Seq gene expression of $T$. dichotomus 598 tissues, organs and developmental samples. Multi-dimensional scaling (MDS) plot showing 599 relatedness between transcript expression profiles of 16 RNA-Seq libraries. Blue circles 600 represent the expression profiles of male samples, and red circles represent those of female 601 samples, while grey circles represent those of samples whose sex are unknown (i.e., embryos). 602 The labels indicating the tissues and sources are defined as follows: Egg, eggs dissected out 603 from the mature ovary; Tt, testis; Ov, ovary; EmM, middle-stage embryos at the stage of ventral 604 appendage formation; EmL1, late stage embryo at the stage of appendages extend; EmL2, late605 stage embryos at the stage of slightly more developed than EmL1; EmL3, late-embryos at a 606 stage of the tracheal pits can be clearly observed; EmL4, late-embryos at a stage of the full607 grown embryo; Mt, Malpighian tubules of third instar larva; Hg, hindgut tube of third instar larva; $608 \mathrm{Fb}$, fat bodies of third instar larva; $\mathrm{Br}$, brains of third instar larvae. See Table S1 for details of the 609 label description. (b) Gene co-expression analysis of the T. dichotomus transcriptome.

610 Hierarchical cluster tree of the $T$. dichotomus genes showing co-expression modules identified 611 using WGCNA. Modules correspond to branches are labelled by colors as indicated by the color 612 band underneath the tree. In total, 13 co-expression modules were identified from the 613 expression data of 16 samples. (c) Co-expression gene modules identified using WGCNA are 614 shown. Each circle represents the value of the respective module's Eigengene. Module names 615 shown above each panel correspond to (b). The sample abbreviations indicated by labels at the 616 bottom of each panel are defined in the legend of (a). The number at the top right in each panel 617 indicates the number of genes belonging to the module specified. The name of organ or 618 developmental stage in which module genes preferentially expressed is displayed at top-right or 619 top-left corner in each panel. 
bioRxiv preprint doi: https://doi.org/10.1101/2022.01.10.475740; this version posted January 12,2022 . The copyright holder for this preprint (which was not certified by peer review) is the author/funder. All rights reserved. No reuse allowed without permission.

a

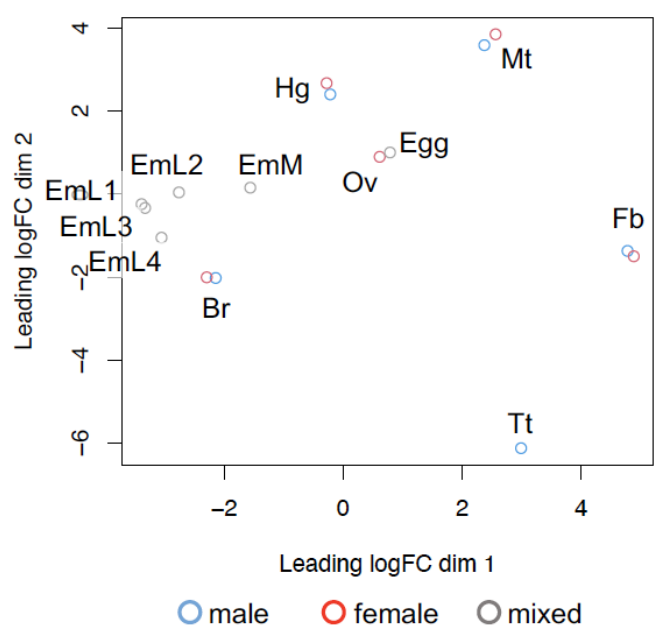

b

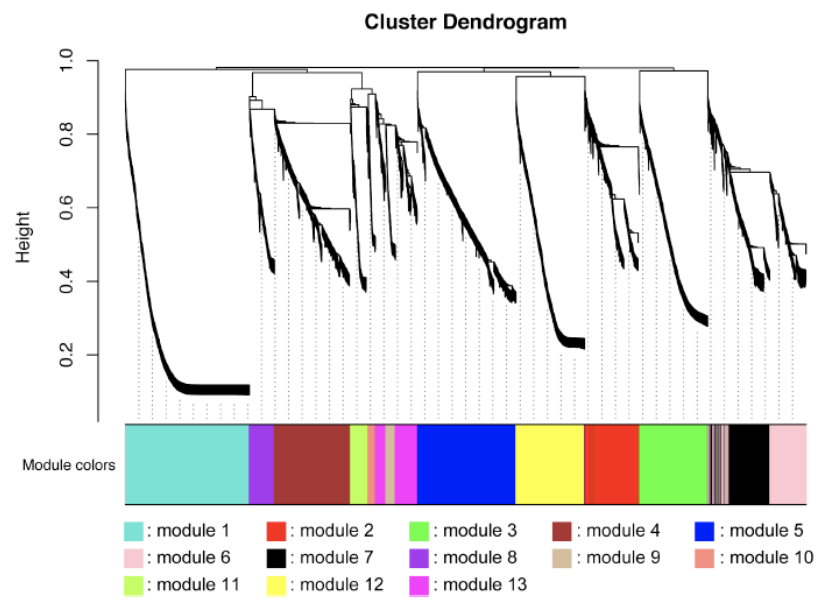

C
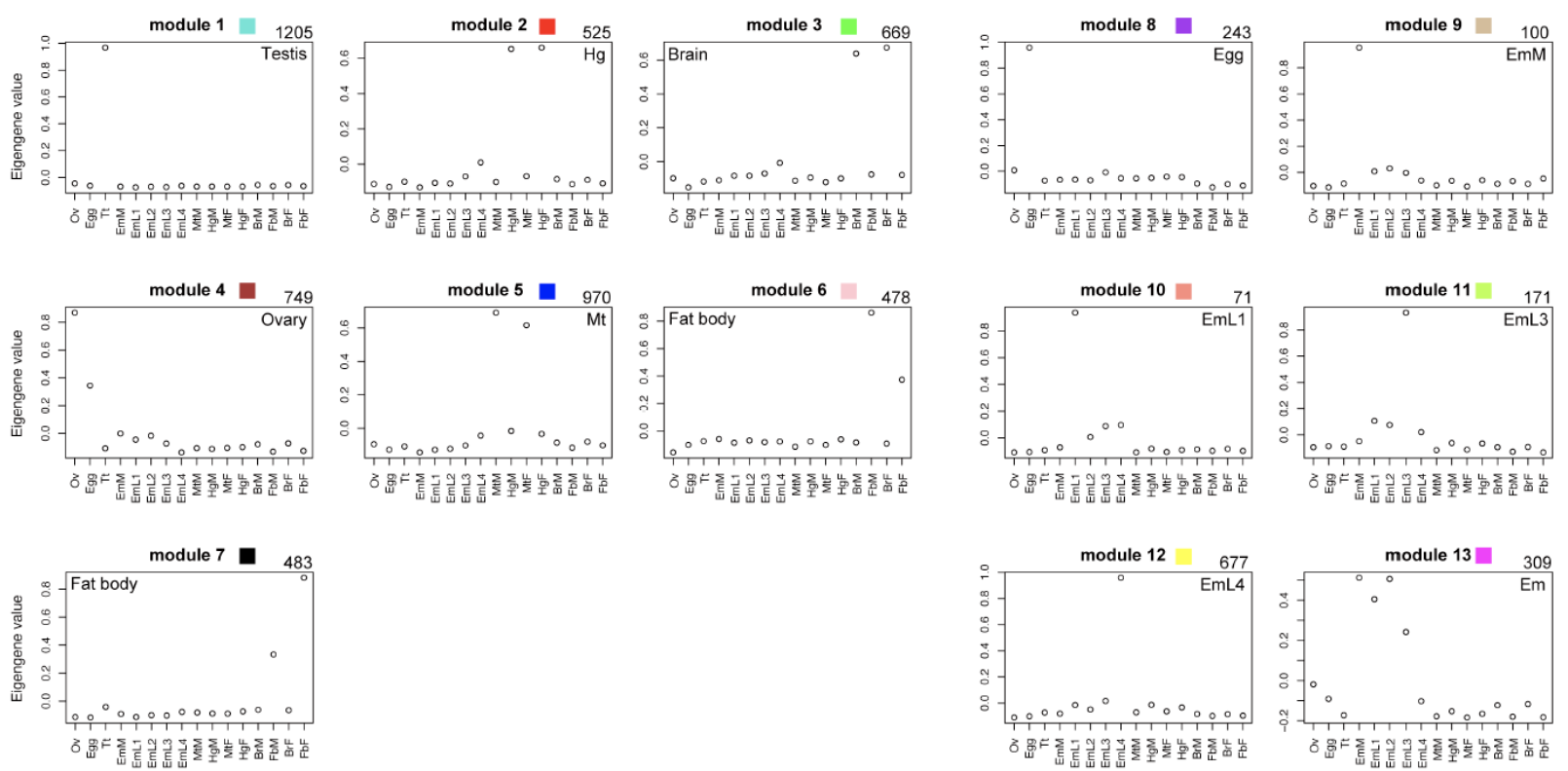


\section{Tables}

622 Table 1 . Summary of $T$. dichotomus genome assembly

\begin{tabular}{llr}
\hline Genome & Assembly size* & $615,027,545$ \\
& No. scaffolds & 15,609 \\
& Scaffold N50 & $8,021,426$ \\
& Longest scaffold & $25,115,014$ \\
& GC\% & 34.8 \\
& No. Ns & $20.0 \mathrm{Mb}$ \\
Annotation & No. coding genes & 23,987 \\
& Repeat content & $305 \mathrm{Mb}(49.5 \%)$ \\
\hline
\end{tabular}

* Genome size estimated with flow cytometry or k-mer distibution is $710-773 \mathrm{Mb}$. (See main texts) 
623 Table 2. BUSCO analysis of the genome assembly of $T$. dichotomus using Insecta gene set.

\begin{tabular}{lcc}
\hline Database & insecta_odb10 & \\
Complete BUSCOs & 1352 & $98.9 \%$ \\
Complete and single-copy BUSCOs & 1335 & $97.7 \%$ \\
Complete and duplicated BUSCOs & 17 & $1.2 \%$ \\
Fragmented BUSCOs & 7 & $0.5 \%$ \\
Missing BUSCOs & 8 & $0.6 \%$ \\
Total BUSCO groups searched & 1367 & \\
\hline
\end{tabular}


bioRxiv preprint doi: https://doi.org/10.1101/2022.01.10.475740; this version posted January 12, 2022. The copyright holder for this preprint (which was not certified by peer review) is the author/funder. All rights reserved. No reuse allowed without permission.

\section{Table S1. Samples used for RNA-seq}

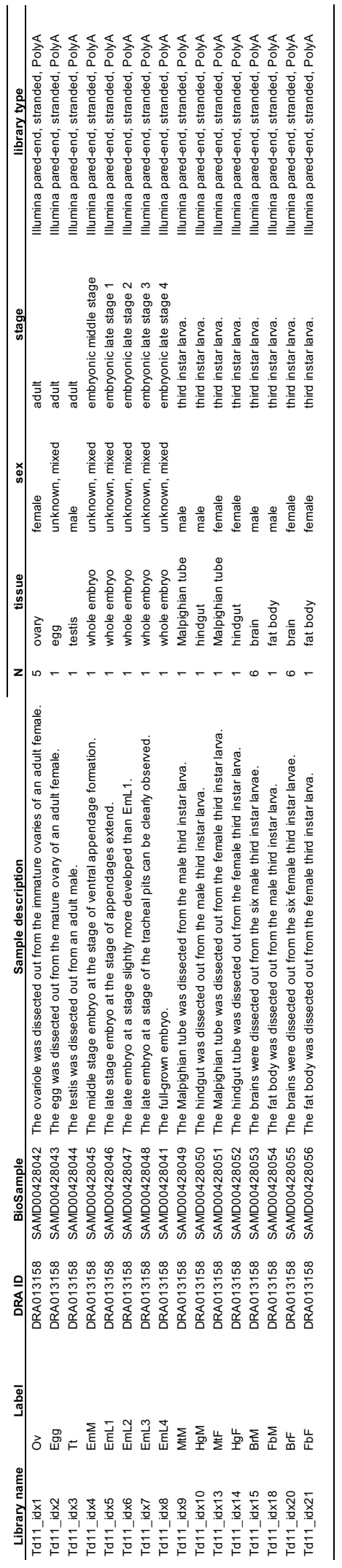


bioRxiv preprint doi: https://doi.org/10.1101/2022.01.10.475740; this version posted January 12, 2022. The copyright holder for this preprint (which was not certified by peer review) is the author/funder. All rights reserved. No reuse allowed without permission.

625 Table S2. Repetitive elements in the T. dichotomus genome.

\begin{tabular}{|c|c|c|c|}
\hline & & Total length (bp) & Percentage (\%) \\
\hline SINES & 6798 & 758637 & 0.12 \\
\hline ALUs & 0 & 0 & 0 \\
\hline MIRs & 2487 & 273122 & 0.04 \\
\hline LINEs & 137006 & 48445463 & 7.88 \\
\hline LINE1 & 0 & 0 & 0 \\
\hline LINE2 & 13502 & 4608407 & 0.75 \\
\hline L3/CR1 & 36384 & 15348005 & 2.5 \\
\hline LTR elements & 22213 & 5196007 & 0.84 \\
\hline ERVL & 0 & 0 & 0 \\
\hline ERVL-MaLRs & 0 & 0 & 0 \\
\hline ERV_classI & 0 & 0 & 0 \\
\hline ERV_classII & 0 & 0 & 0 \\
\hline DNA elements & 503264 & 176254643 & 28.66 \\
\hline hAT-Charlie & 10248 & 2803595 & 0.46 \\
\hline TcMar-Tigger & 6485 & 2377631 & 0.39 \\
\hline Unclassified: & 337209 & 70318994 & 11.43 \\
\hline Total interspersed repeats & & 300973744 & 48.94 \\
\hline Small RNA: & 1229 & 194443 & 0.03 \\
\hline Satellites: & 0 & 0 & 0 \\
\hline Simple repeats: & 69943 & 3380637 & 0.55 \\
\hline Low complexity: & 10747 & 543899 & 0.09 \\
\hline Total repeat bases & & 304697749 & 49.54 \\
\hline
\end{tabular}

* most repeats fragmented by insertions or deletions have been counted as one element 
626 Data sets at FigShare

627 (https://doi.org/10.6084/m9.figshare.c.5737754)

628 - Genome assembly: doi: 10.6084/m9.figshare.17126303

629 - Gene model: doi: 10.6084/m9.figshare.17126306

630 - Gene model annotation: 10.6084/m9.figshare.17126312

631 - RNA-seq data: 10.6084/m9.figshare.17126315

632 - RNA-seq WGCNA analysis: doi: 10.6084/m9.figshare.17126327

633 